\title{
GYNAECOLOGICAL CANCER
}

\section{Biomarker potential of CA-125 enhanced}

Serum CA-125 is a promising diagnostic biomarker for ovarian cancer, but screening studies using fixed CA-125 cutoffs have not shown a mortality benefit in the general (low-risk) population. New data suggest that risk-based triage of screening assessments according to the trajectory of serum CA-125 levels could be more effective.

Usha Menon et al. performed 296,911 annual CA-125 screens among 46,237 women in the multimodal screening (MMS) arm of the UKCTOCS. The Risk of Ovarian Cancer Algorithm (ROCA) was used to determine the frequency and type of follow-up assessment. "The ROCA is essentially a Bayesian statistical formula that uses age and CA-125 pattern measured over time to calculate the likelihood of a woman being diagnosed with ovarian cancer in the year following the test," explains Menon.

Overall, 155 of the 46,237 women were diagnosed with invasive epithelial ovarian cancer (iEOC) within 1 year of screening. "The key message is that the protocol adopted in the UKCTOS MMS arm detected $86 \%$ of women with iEOC.
If CA- 125 cutoffs of $35 \mathrm{U} / \mathrm{ml}$ or $30 \mathrm{U} / \mathrm{ml}$ were used, only $41 \%$ or $48 \%$, respectively, of the women with screen-detected cancers would have been picked up at their annual screen," states Menon. Ovarian cancer can be associated with low but rising serum CA-125 levels, whereas some benign conditions result in high but static CA-125 levels; thus, 'CA- 125 velocity' improved the sensitivity and specificity of ovarian cancer detection. "Serial biomarker measurements may be much more informative than fixed cutoffs in the context of cancer screening and should be considered for other cancers."

"It remains unknown whether early detection of these cancers will translate into lives saved," Menon concludes; mortality data from the UKCTOCS MMS and control arms are due later this year.

\section{David Killock}

Original article Menon, U. et al. Risk algorithm using serial biomarker measurements doubles the number of screendetected cancers compared with a single-threshold rule in the United Kingdom Collaborative Trial of Ovarian Cancer Screening. J. Clin. Oncol. doi:10.1200/JC0.2014.59.4945 